\title{
Within-Host Models of High and Low Pathogenic Influenza Virus Infections: The Role of Macrophages
}

\author{
Kasia A. Pawelek ${ }^{1 *}$, Daniel Dor, $\mathrm{Jr}^{2}{ }^{2}$, Cristian Salmeron ${ }^{1}$, Andreas Handel $^{3 *}$ \\ 1 Department of Mathematics and Computational Science, University of South Carolina Beaufort, Bluffton, \\ South Carolina, United States of America, 2 Department of Natural Sciences, University of South Carolina \\ Beaufort, Bluffton, South Carolina, United States of America, 3 Department of Epidemiology and \\ Biostatistics, College of Public Health, University of Georgia, Athens, Georgia, United States of America \\ *kpawelek@uscb.edu (KAP); ahandel@uga.edu (AH)
}

\section{G openaccess}

Citation: Pawelek KA, Dor D, Jr., Salmeron C, Handel A (2016) Within-Host Models of High and Low Pathogenic Influenza Virus Infections: The Role of Macrophages. PLoS ONE 11(2): e0150568. doi:10.1371/journal.pone.0150568

Editor: Jie Sun, Indiana University, UNITED STATES

Received: September 28, 2015

Accepted: February 14, 2016

Published: February 26, 2016

Copyright: @ 2016 Pawelek et al. This is an open access article distributed under the terms of the Creative Commons Attribution License, which permits unrestricted use, distribution, and reproduction in any medium, provided the original author and source are credited.

Data Availability Statement: The data used in the study is published in the following peer-reviewed journal: Perrone LA, Plowden JK, Garcia-Sastre A, Katz JM, Tumpey TM (2008) H5N1 and 1918 pandemic influenza virus infection results in early and excessive infiltration of macrophages and neutrophils in the lungs of mice. PLoS Pathog 4: e1000115.

Funding: This publication was partially supported by a grant from the National Institute of General Medical Sciences, P20GM103499, SC INBRE (KAP and DD). The research of CS was partially supported by the University of South Carolina Mini Grant Program and SC EPSCoR/IDeA Scientific Advocate Network. AH was partially supported by the National Institute of

\section{Abstract}

The World Health Organization identifies influenza as a major public health problem. While the strains commonly circulating in humans usually do not cause severe pathogenicity in healthy adults, some strains that have infected humans, such as $\mathrm{H} 5 \mathrm{~N} 1$, can cause high morbidity and mortality. Based on the severity of the disease, influenza viruses are sometimes categorized as either being highly pathogenic (HP) or having low pathogenicity (LP). The reasons why some strains are LP and others HP are not fully understood. While there are likely multiple mechanisms of interaction between the virus and the immune response that determine LP versus HP outcomes, we focus here on one component, namely macrophages (MP). There is some evidence that MP may both help fight the infection and become productively infected with HP influenza viruses. We developed mathematical models for influenza infections which explicitly included the dynamics and action of MP. We fit these models to viral load and macrophage count data from experimental infections of mice with LP and HP strains. Our results suggest that MP may not only help fight an influenza infection but may contribute to virus production in infections with HP viruses. We also explored the impact of combination therapies with antivirals and anti-inflammatory drugs on HP infections. Our study suggests a possible mechanism of MP in determining HP versus LP outcomes, and how different interventions might affect infection dynamics.

\section{Introduction}

The World Health Organization (WHO) identifies influenza as a major public health problem [1]. Every year people get infected with seasonal, zoonotic, or pandemic strains of influenza. Influenza strains can be categorized as having either low pathogenicity (LP) or high pathogenicity (HP), which refers to the ability of the virus to induce disease in a specific host. Infections with HP avian influenza have led to severe complications in children and young adults [2,3]. A recent outbreak of an H7N9 avian influenza strain occurred in China during the spring of 2013 
Allergy and Infectious Diseases of the National Institutes of Health under award number U19Al117891. The content is solely the responsibility of the authors and does not necessarily represent the official views of the funders. The funders had no role in study design, data collection and analysis, decision to publish, or preparation of the manuscript.

Competing Interests: The authors have declared that no competing interests exist. and was reported to have caused 135 human cases and 44 deaths [4]. The most catastrophic influenza related pandemic, caused by an $\mathrm{H} 1 \mathrm{~N} 1$ strain with an increased pathogenicity (several times the mortality of typical seasonal strains), occurred in 1918 and caused approximately 500 million infection cases and an estimated 50-100 million deaths [5]. Infections with low pathogenicity influenza viruses are less likely to cause severe illness or lead to the death of the infected individual. A better understanding of the mechanisms that may lead to severe infections caused by the HP viruses would be very valuable in our continued efforts to combat influenza.

Because of the limitations associated with data collection during human HP influenza infections, animal experiments and models are essential in obtaining a better understanding of viral load regulation within a host. Numerous mammalian models have been examined to investigate avian influenza development; they have provided us with crucial information about the disease [6-10]. Complementing these experimental studies, mathematical models can provide insight into understanding infection dynamics and the role of immune response in controlling the disease or leading to complications in certain cases of the disease.

A number of mathematical models have been developed to study the dynamics of uncomplicated influenza virus infection and immune responses [11-21]. A recent modeling study showed that a model with two types of susceptible cells could explain elevated viral titer in HP infections and provide a more realistic fit to HP viral load data than a model with one cell type [22]. However, it was not specified what type of cells were represented by the second cell population in the model.

Here, we further explore this idea of a secondary population of cells that can be productively infected. We specifically focus on the role of macrophages. Macrophages constitute an important component of the innate immune response and have been shown to have an important role during influenza infections [23-27]. However, their role in HP influenza infections is still uncertain.

In [28] it was shown that in fatal infections with HP H1N1 and H5N1 influenza viruses high numbers of macrophages and neutrophils are expressed in the lungs. This study also performed experiments in vitro, showing that primary macrophages and dendritic cells are susceptible to HP virus infection [28]. Other studies have also shown that macrophages can be productively infected with influenza viruses [29, 30]. In [29], it was shown that the H5N1 virus can productively replicate in alveolar macrophages. Furthermore, primary human macrophages infected with avian $\mathrm{H} 5 \mathrm{~N} 1$ resulted in more efficient productive replication than infection with human influenza viruses [30]. The viral replication competence of macrophages and their contribution to overall functions in the pathogenesis of the infection with HP viruses are not fully understood. In our modeling study we explored protective and pathogenic functions of macrophages and highlight their possible role as cells that contribute both to immune response function and virus production. After showing that our model can capture the dynamics of HP infections, we use our model to explore the impact of drugs on HP infection dynamics.

\section{Materials and Methods}

\section{Mathematical model}

We developed a mathematical model based on differential equations to study the within-host dynamics of influenza infection. The model has seven variables: uninfected epithelial cells susceptible to infection $(T)$, productively infected epithelial cells $(I)$, free virus $(V)$, uninfected resting macrophages circulating within the host $\left(M_{R}\right)$, activated macrophages at the site of the 
infection $\left(M_{A}\right)$, productively infected macrophages $\left(M_{I}\right)$, and the antibody/B-cell component of the immune response $(A)$.

A schematic diagram of the model is shown in Fig 1. Variables and parameters are summarized in Tables 1 and 2, respectively. The mathematical formulation of the model is given by the following set of ordinary differential equations:

$$
\begin{aligned}
& \frac{\mathrm{d} T}{\mathrm{~d} t}=-\beta T V \\
& \frac{d I}{d t}=\beta T V-\delta_{I} I \\
& \frac{d V}{d t}=\left(1-\varepsilon_{1}\right)\left(p I+p_{M} M_{I}\right)-c V-\kappa A V-\beta T V \\
& \frac{d M_{R}}{d t}=s-\left(1-\varepsilon_{2}\right) \alpha V M_{R} /\left(V_{50}+V\right)-\delta_{M R} M_{R} \\
& \frac{d M_{A}}{d t}=\left(1-\varepsilon_{2}\right) \alpha V M_{R} /\left(V_{50}+V\right)-\gamma M_{A} V-\delta_{M A} M_{A} \\
& \frac{d M_{I}}{d t}=\gamma M_{A} V-\delta_{M I} M_{I} \\
& \frac{d A}{d t}=\mu M_{A}+\rho A
\end{aligned}
$$

Infection of susceptible epithelial cells is described by the term $\beta V T$, which represents the rate of encounter with virus and subsequent infection. Infected cells die at a rate $\delta_{I}$. Virus particles are produced by infected epithelial cells at rate $p$. Additional production of virus by infected macrophages occurs at rate $p_{M}$. Virus is cleared by the B-cell/antibody immune response at rate $\kappa A V$. Additional, non-antibody specific virus clearance occurs at the rate $c$.

Non-activated macrophages $\left(M_{R}\right)$ are assumed to be generated at the constant rate $s$ and to die at rate $\delta_{M R}$ [31]. The term $\alpha V M_{R} /\left(V_{50}+V\right)$ represents the rate at which macrophages are activated. This activation is assumed to be proportional to the viral load, with a maximum activation at the rate $\alpha$, the parameter $V_{50}$ represents the viral load at which activation reaches half its maximum. The activated macrophages at the site of infection $\left(M_{A}\right)$ help activate the adaptive immune response $[24,26]$. Mechanistically, this activation is likely indirect, with MP producing pro-inflammatory cytokines and chemokines, which in turn help activate other cells of the innate response, e.g. dendritic cells, and the adaptive response. To keep our model simple, we describe this indirect, multi-step interaction from MP to adaptive response by a direct activation rate, which we model to occur proportional to the number of activated MP at rate $\mu M_{A}$.

Activated macrophages die at rate $\delta_{M A}$ and, in the case of infection with HP viruses, activated macrophages at the site of the infection can be infected [28-30], which we model to occur at rate $\gamma M_{A} V$. Infected macrophages are assumed to lose their ability to help activate the adaptive immune response and instead start producing virus [28-30] at rate $p_{M}$. Infected macrophages die at rate $\delta_{M I}$. B-cells that have been activated proportional to the number of macrophages grow exponentially through division at rate $\rho$. Since we are only interested in the acute infection dynamics, we do not model contraction of the adaptive immune response after clearance of the infection.

B-cells/antibodies clear virus particles [32], which in our model occurs at the rate $\kappa A V$. Note that we simply assume that antibodies are proportional to B-cells and therefore do not use two separate equations for B-cells and antibodies but instead combine them in one equation. 


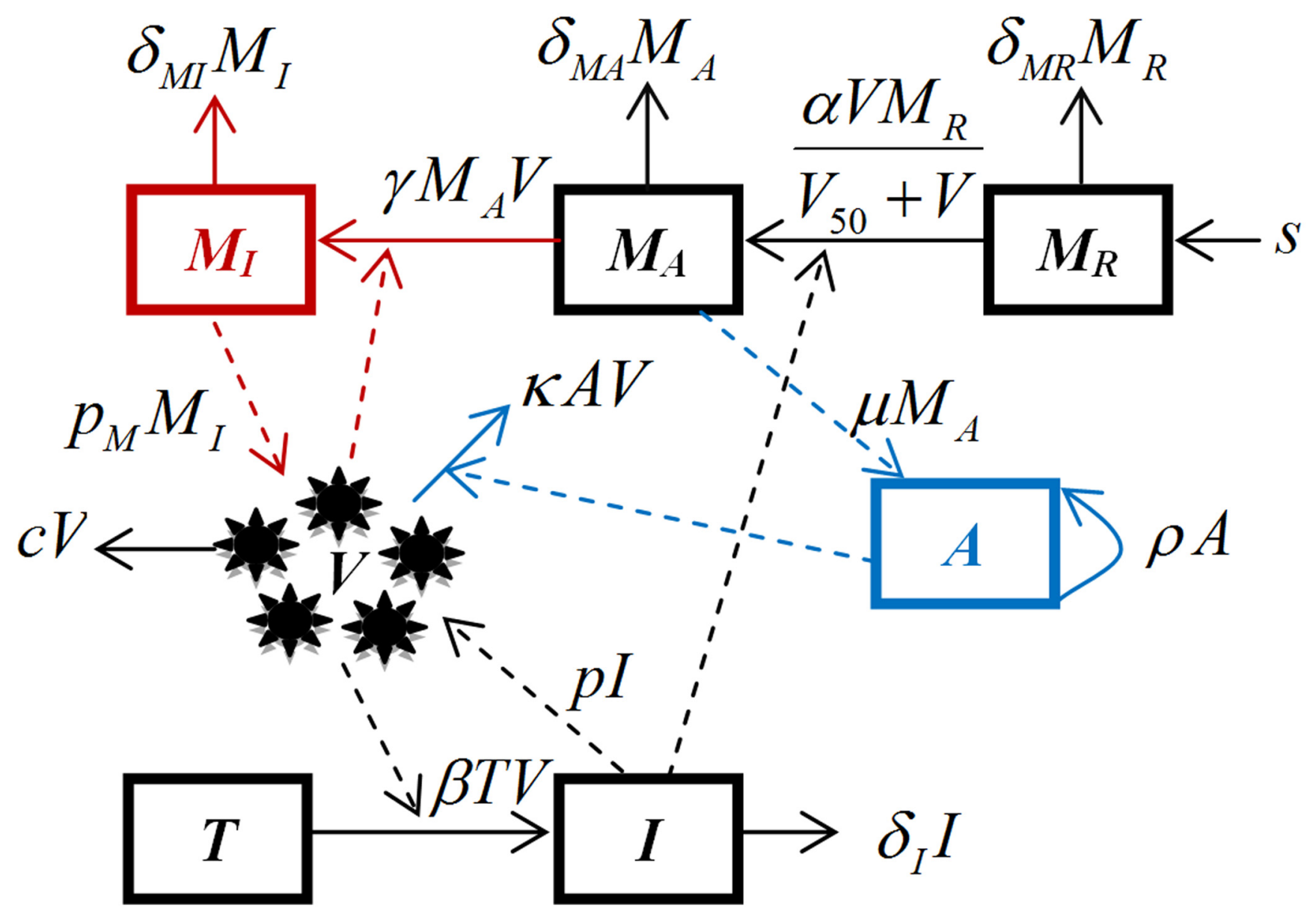

Fig 1. Schematic representation of the full model. A detailed description of the model, the set of differential equations, and meaning and values for variables, and parameters are given in the Materials and Methods section and Tables 1 and 2.

doi:10.1371/journal.pone.0150568.g001

Our hypothesis is that LP and HP infections differ, in part, due to HP viruses' capability to productively infect macrophages. To show this in our model, we run simulations for the LP scenarios with parameters $\gamma$ and $p_{M}$ set to zero, i.e. no infection of and subsequent virus production by macrophages occurs. In contrast, for the HP scenarios, these parameters are allowed to be non-zero.

We also investigate the effect of two different potential treatments that might be given to combat HP influenza infections. In particular, we incorporated the effect of neuraminidase

Table 1. Definitions of variables used in the model and their initial values.

\begin{tabular}{lll}
\hline Variable & Definition & Initial Condition \\
\hline$T$ & Uninfected epithelial cells susceptible to infection & $7 \times 10^{9},[12]$ \\
$I$ & Infected epithelial cells & 0 \\
$M_{R}$ & Uninfected macrophages circulating the host & $s / \delta_{M R},[31]$ \\
$M_{A}$ & Activated macrophages at the infection site & 0 \\
$M_{I}$ & Productively infected macrophages & 0 \\
$V$ & Virus & $1 \times 10^{2}$, as reported in [28] \\
$A$ & Immune response due to antibodies & 0 \\
\hline
\end{tabular}

doi:10.1371/journal.pone.0150568.t001 
Table 2. Parameter definitions, units, values, and references.

\begin{tabular}{|c|c|c|c|}
\hline Symbol & Definition & Unit & Value and Reference \\
\hline$\delta_{M R}$ & Death rate of uninfected macrophages, $M_{R}$ & day $^{-1}$ & $1 / 25$, mean based on [31] \\
\hline$\delta_{M A}$ & Death rate of activated macrophages at the infection site, $M_{A}$ & day $^{-1}$ & see text \\
\hline$\delta_{M I}$ & Death rate of infected macrophages, $M_{I}$ & day $^{-1}$ & see text \\
\hline$s$ & Constant generation rate of uninfected macrophages circulating within a host $\left(M_{R}\right)$ & cells day ${ }^{-1}$ & fitted \\
\hline$\delta_{l}$ & Death rate of infected epithelial cells & day $^{-1}$ & $2,[10,20]$ \\
\hline$\beta$ & Infection rate of epithelial cells & $(\mathrm{PFU})^{-1} \mathrm{ml} \mathrm{day}^{-1}$ & fitted \\
\hline$\alpha$ & Maximum recruitment rate of macrophages to the site of infection & day $^{-1}$ & fitted \\
\hline$V_{50}$ & Virus load at which activation reaches half its maximum & $(\mathrm{PFU})^{-1} \mathrm{ml}$ & fitted \\
\hline y & Infection rate of macrophages & $(\mathrm{PFU})^{-1} \mathrm{ml} \mathrm{day}^{-1}$ & fitted \\
\hline$p$ & Virus production rate from epithelial cells & $\operatorname{PFU}(\mathrm{ml})^{-1}$ day $^{-1}$ cell $^{-1}$ & fitted \\
\hline$p_{M}$ & Virus production rate from macrophages & PFU $(m l)^{-1}$ day $^{-1}$ cell $^{-1}$ & fitted \\
\hline$\mu$ & Activation of immune response & day $^{-1}$ & fitted \\
\hline$\rho$ & Rate of expansion of B-cells & day $^{-1}$ & $1,[12]$ \\
\hline$\kappa$ & Clearance rate of free virus due to immune system & day $^{-1}$ cell $^{-1}$ & fitted \\
\hline$c$ & Virus clearance rate due to mechanisms other than antibodies & day $^{-1}$ & $3,[11,13]$ \\
\hline$\varepsilon_{1}$ & Antiviral treatment efficacy & - & $0-1$ \\
\hline$\varepsilon_{2}$ & Anti-inflammatory treatment efficacy & - & $0-1$ \\
\hline
\end{tabular}

doi:10.1371/journal.pone.0150568.t002

inhibitors (oseltamivir and zanamivir), which are widely used against influenza infection [33]. Similarly to previous modeling studies $[13,34]$ we introduce the antiviral effect of the neuraminidase by lowering the viral production by a factor of $\left(1-\varepsilon_{1}\right)$, where $\varepsilon_{1}$ is the drug efficacy. We further model the effect of an anti-inflammatory drug which inhibits the activation and recruitment of macrophages to the site of infection, with efficacy $\varepsilon_{2}$.

\section{Experimental data}

We compared our model to data from experimental influenza infection studies of $\mathrm{BALB} / \mathrm{c}$ mice infected with LP viruses: TX/91 (H1N1) and SP/83 (H5N1) and HP viruses: 1918 (H1N1) and Thai/16 (H5N1) [28]. Specifically, viral load and macrophage data were extracted from Figs 1 and 2 in [28] using Engauge Digitizer (digitizer.sourceforge.net). For further details about the data see the original study.

It is worth noting that the markers used in [28] to identify macrophages might not have captured all subtypes of macrophages and further might include subpopulations of cells that are not classically defined as macrophages. The difficulty of cleanly defining and counting macrophages based on specific markers is a limitation of the available data. For our study purposes we assume that the measured cells represent the bulk of the activated macrophages. However, this caveat with regard to the experimental data needs to be kept in mind.

\section{Parameter values and data fitting}

To avoid over fitting of the models, some of the parameters were fixed, with values taken from the existing literature. The lifespan of infected epithelial cells, $1 / \delta_{I}$, was fixed at 0.5 days in agreement with previous modeling studies [10, 20,35]. The initial population of epithelial cells in the mice lungs was fixed at $7 \times 10^{9}$ cells based on a value provided in [12]. We set the initial population of infected epithelial cells and infected macrophages to 0 . Following [31], we set the initial number of uninfected, resting macrophages to $M_{R}(0)=s / \delta_{M R}$. Death rates of macrophages in the different states, $\left(\delta_{M R}, \delta_{M A}\right.$, and $\left.\delta_{M I}\right)$, are assumed to be equal due to the lack of 
data and their value is taken to be $1 / 25$ day $^{-1}$ based on [31]. Virus clearance rate due to mechanisms other than antibodies, $c$, was set to $3 \mathrm{day}^{-1}$, following [11, 13]. The rate of expansion of B-cells, $\rho$, was set to 1 day $^{-1}$, based on [12].

The remaining parameters were estimated by fitting the model to the data described above. Specifically, for the H1N1, as well as, the H5N1 viruses studied in [28], we fit viral load and macrophage data for the LP and HP strains simultaneously to the same model, with the difference being that the parameters describing macrophage infection and virus production ( $\gamma$ and $p_{M}$, respectively) are zero for LP and non-zero for HP. Additionally, LP and HP strains were allowed to vary in their rate of activation of macrophages, $\alpha$. All other parameter values are shared between the LP and HP scenarios. This allows us to test our hypothesis that differential activation and productive infection of macrophages can explain the observed differences between LP and HP infections.

To allow simultaneous fitting of two different experimental quantities, namely viral load and macrophage numbers, we follow [21] and fit the model by minimizing the weighted sum of square differences, with weights used to standardize viral load and macrophage contributions and allow for joint summation. The objective function that we minimize is given by the following equation:

$$
S S R=\sum_{i=1}^{n_{V}}\left(\frac{\log _{10} V_{i}^{m}-\log _{10} V_{i}}{\log _{10} V_{\max }}\right)^{2}+\sum_{i=1}^{n_{M}}\left(\frac{\log _{10}\left(M_{A}^{m}+M_{I}^{m}\right)_{i}-\log _{10} M_{i}}{\log _{10} M_{\max }}\right)^{2}
$$

Where viral load data (for both LP and HP strains) is given by $V_{i}$ and the corresponding value predicted by the model is $V_{i}^{m}$. Macrophage data is given by $M_{i}$ and the analogous model prediction for the sum of the activated and infected macrophages at the site of the infection is represented by $\left(M_{A}{ }^{m}+M_{I}{ }^{m}\right)_{i}$. The maximum data values of the viral load and macrophages are denoted by $V_{\max }$ and $M_{\max }$, respectively.

To deal with viral load data that is at or below the limit of detection (left-censored), we keep the squared difference if the model predicts a value above the limit of detection, but set any difference to zero for a model prediction that is below the limit of detection $\left(10^{0.5} \mathrm{PFU} /\right.$ $\mathrm{ml})$. Lastly, since the reported data did not track the infection all the way to its conclusion, we augmented the data by adding a value for the virus load at the limit of detection 15 days post infection. This was required to ensure fits that agree with the known biology of the infection dynamics.

We used R Version 3.2. [36], as well as, the packages nloptr [37] and deSolve [38] to fit the model to the data. Packages dplyr [39] and ggplot2 [40] were also used. The data and R scripts to reproduce all results are provided as supplementary material.

\section{Results}

\section{Productive infection of macrophages can explain HP infection dynamics}

Dobrovolny et. al. showed that the cell tropism may explain the observed disease severity of influenza infections caused by HP viruses [22]. Their model included two cell populations, which have different susceptibility to the infection and virus production rates, and was fitted to viral load data [22]. While [22] envisioned these two cell types to be different types of epithelial cells, here we consider the possibility that the second cell type are macrophages, which can both become productively infected and also play a role in combating the infection. We fit the model to both viral load and macrophage data and explore the hypothesis that macrophages may have both protective and pathogenic role in an HP influenza infection. To test our hypothesis we simultaneously fitted our model to LP and HP infection data, with the only difference 
being that the parameters describing macrophage activation, infection, and virus production $\left(\alpha, \gamma\right.$, and $p_{M}$, respectively) differ between LP and HP, with the latter two being zero for LP. Such a model provides a reasonable fit to the data (Fig 2). This suggests that our hypothesis that the productive infection of macrophages can explain the observed differences between LP and HP infections is plausible.

Furthermore, our modeling predictions suggest that macrophages become activated quickly in both LP and HP infections (Fig 3). In particular, HP infections lead to greater activation of MP. However, the majority of these activated MP become infected (Fig 3), and therefore are not able to properly participate in the immune response, subsequently leading to the increased viral load seen in the data (Fig 2).

As can be seen in Fig 4, our model predicts that the decline in target cells is not considerable. This agrees with experimental evidence of influenza infections, which have shown that -at least in mice-destruction of approximately more than $10 \%$ of alveolar type I cells leads to severe pathology and host death [41]. Models which do not include immune response and where removal of target cells is the only mechanism by which an infection can end predict depletion of target cells beyond biologically reasonable levels, a feature that has been discussed previously [20,42].

\section{Impact of HP model parameters on viral load and macrophage response}

We performed a sensitivity analysis to look more closely at the 3 parameters associated with HP infections in our model, namely $\alpha, \gamma$, and $p_{M}$. For both H1N1 and H5N1 strains, we simulated the model and varied each of these 3 parameters individually in a range of 0.01-100 times the original parameter obtained from the best fit (Table 3). All other parameters were kept at their best fit estimates for the HP strain. For each run, we computed two measures to summarize the infection. As one measure, we followed $[43,44]$ and used total viral load (area under curve). As a second measure, we computed the total number of activated macrophages during the infection. The latter quantity can be thought of as representing a proxy for the amount of inflammation/immune pathology present during the infection. As Fig 5 shows, increased virus production by MP (increased $p_{M}$ ) leads to a higher viral load (Fig 5A and 5C), but as expected, does not impact the number of activated MP (Fig 5B and 5D). The rate at which MP become infected $(\gamma)$ has a similar effect; however has less effect on the viral load increase than the result of the increase of the parameter $p_{M}$ (Fig $5 \mathrm{~A}$ and $5 \mathrm{C}$ ). The only parameter that affects the number of MP is the rate of MP activation ( $\alpha$ ) (Fig 5B and 5D). This rate has little impact on total viral load, with initial increases in MP activation leading to reduced viral load, up to some level after which activation rate has limited further impact.

\section{Antiviral and anti-inflammatory therapies in infections with HP viruses}

We added the effect of two drugs to our model to study how either antiviral or anti-inflammation treatment, or the combination of the two, would affect the infection dynamics. Fig $6 \mathrm{~A}$ and 6C shows that treatment with antivirals, such as neuraminidase inhibitors, leads to a reduction of viral load, which is in line with both experimental data and previous modeling studies [13, 34]. However, for most treatment efficacies, the antiviral drug has little impact on activation of macrophages (Fig 6B and 6D), which we consider here a proxy for immune mediated inflammation and morbidity. This is somewhat reminiscent of observations from treatment of regular (non HP) influenza with neuraminidase antivirals in humans, where there is a clear impact on viral load but relatively modest impact on symptoms [36, 45-47]. Our model predicts that only at efficacies $>90 \%$ the suppression of viral load leads to a subsequent reduction in macrophage activation. In contrast, treatment targeting macrophage activation has essentially no impact on 

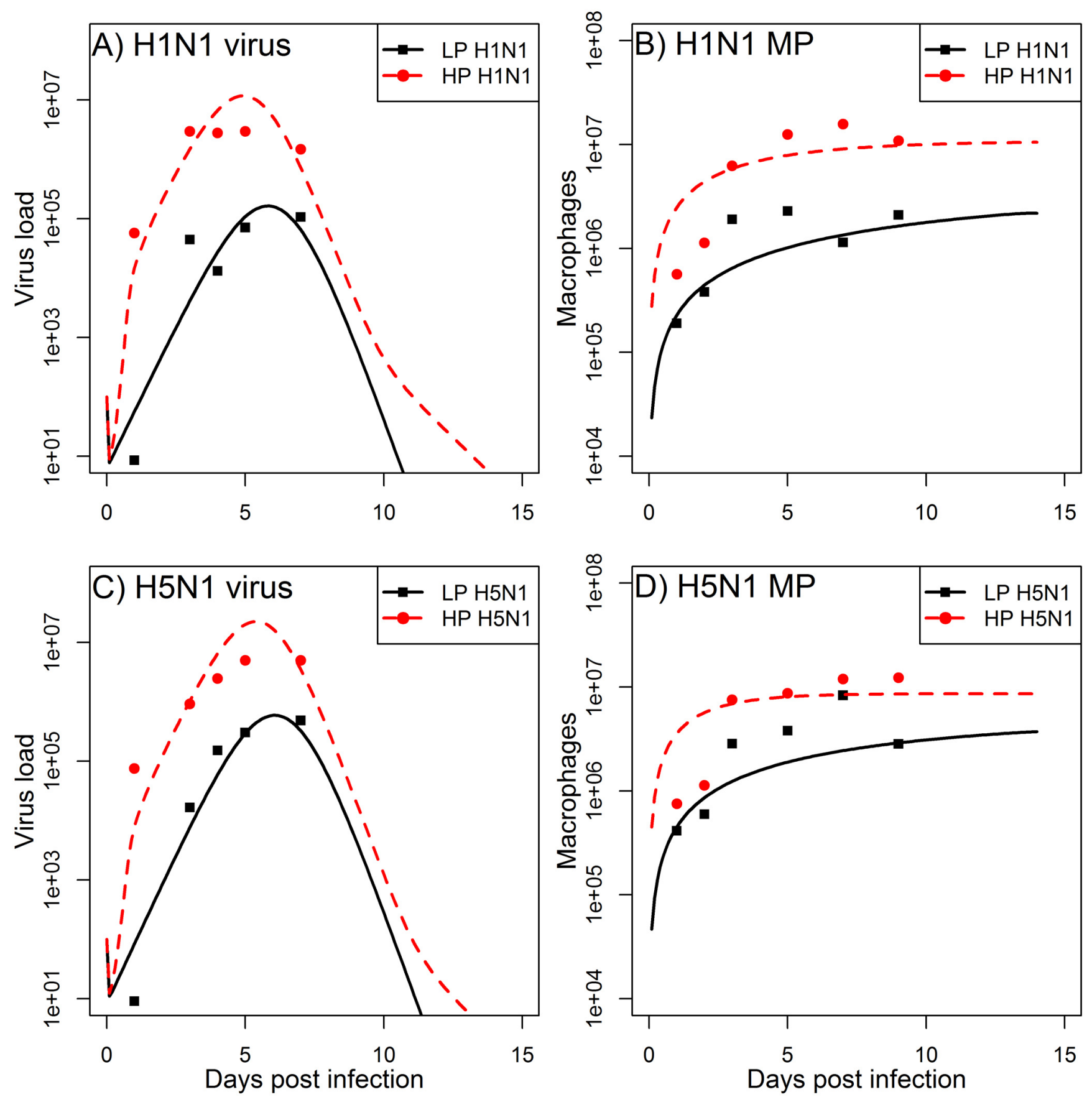

Fig 2. Best fits of the model to the experimental data for viral load and macrophages reported in [28]. Macrophages as predicted by the model are $M_{A}+M_{l}$. Parameter values for the best fit estimates are listed in Tables 2 and 3.

doi:10.1371/journal.pone.0150568.g002

viral load (Fig 6A and 6C), but reduces the total number of activated macrophages even at intermediate values of treatment effectiveness (Fig 6B and 6D). The combination of both drug treatments works additively, with the antiviral reducing viral load and the anti-macrophage activation reducing total number of activated macrophage. Our model suggest that this combined treatment approach, which has been suggested previously [48, 49], seems to be the most promising in overall targeting HP influenza infections. 

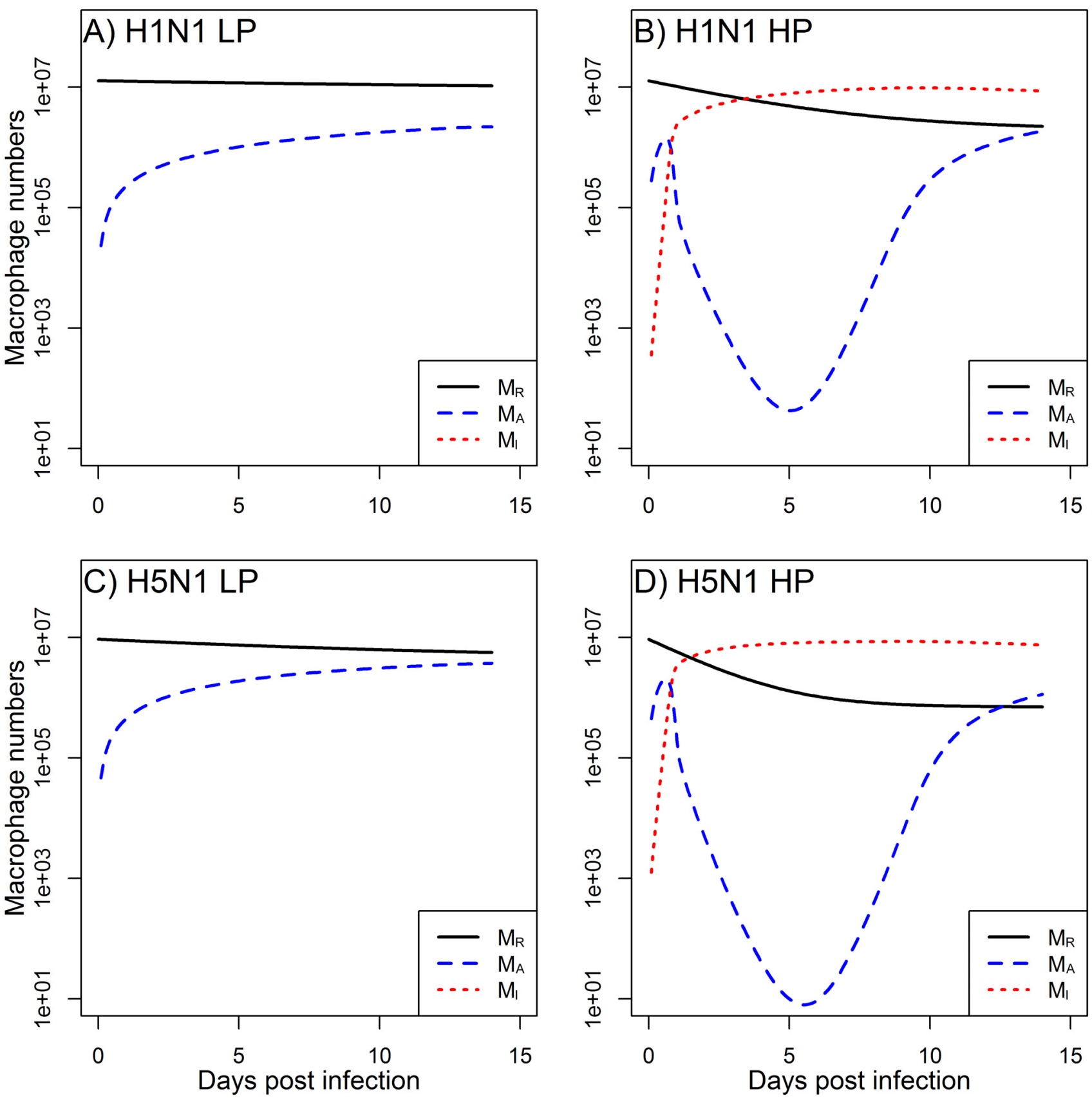

Fig 3. Model predictions for non-activated macrophages $\left(M_{R}\right)$, activated macrophages $\left(M_{A}\right)$, and productively infected macrophages $\left(M_{l}\right)$. Figure shows the LP and HP H1N1 and H5N1 scenarios corresponding to the viral load and total macrophage model results shown in Fig 2. Parameter values are listed in Tables 2 and 3.

doi:10.1371/journal.pone.0150568.g003

We considered here a very optimistic scenario where treatment occurs 1 day post infection. Clinically, this is hard to achieve. Obviously, a later start of treatment reduces the impact of both interventions. Starting treatment 2 days post infection or later leads to little impact on virus load and macrophage numbers, even at high efficacies (simulations not shown). This agrees with the general understanding that rapid intervention post infection is crucial for maximum impact. 

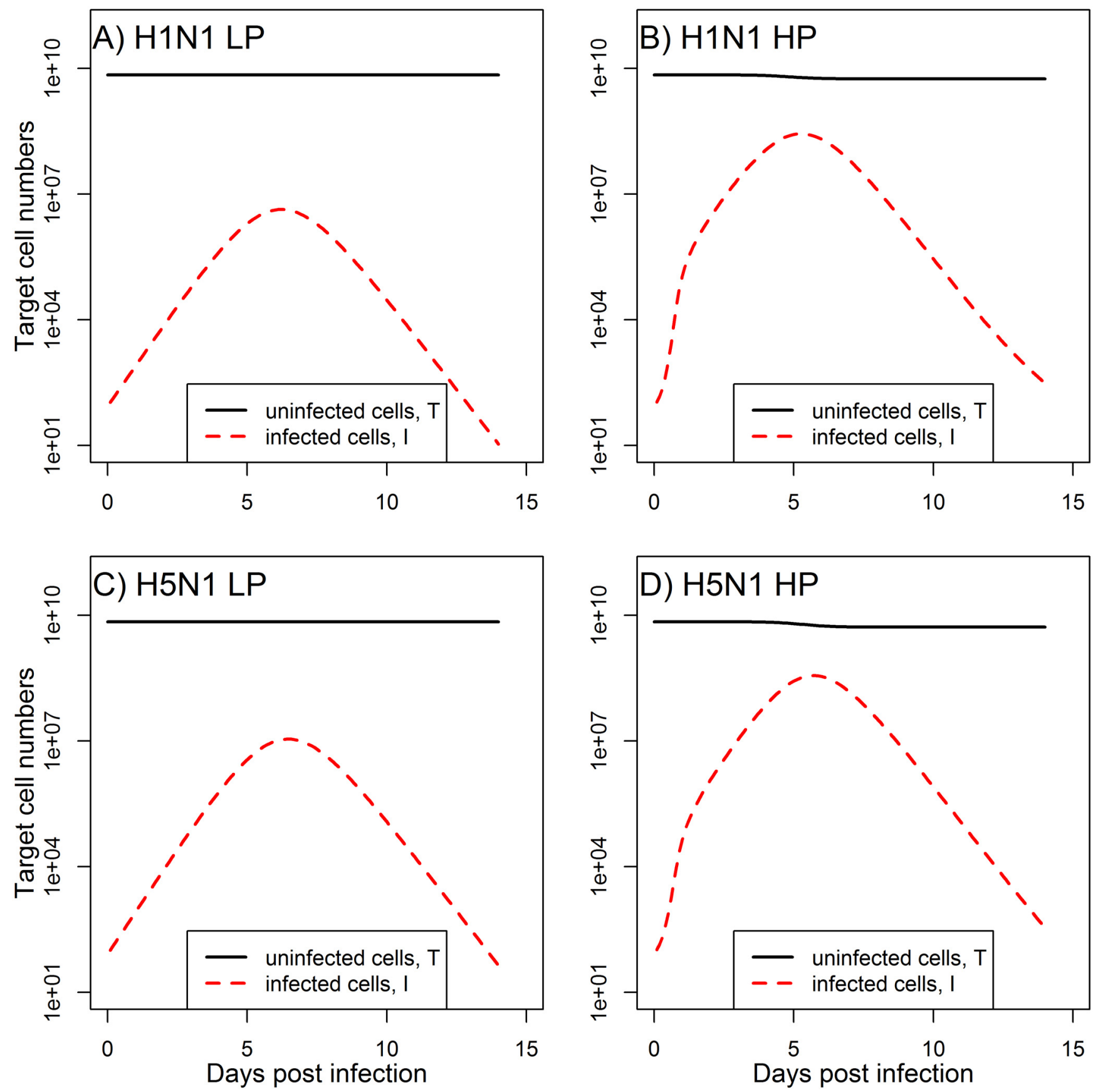

Fig 4. Model predictions for the susceptible epithelial cells $(\boldsymbol{T})$ and infected epithelial cells $(\boldsymbol{I})$. The figure shows the LP and HP H1N1 and H5N1 scenarios corresponding to the viral load and macrophage model results shown in Figs 2 and 3. Parameter values are listed in Tables 2 and 3.

doi:10.1371/journal.pone.0150568.g004

Table 3. Best fit parameter values to the viral load and macrophage data. The best fits are displayed in Fig 2.

\begin{tabular}{|c|c|c|c|c|c|c|c|c|c|c|}
\hline Virus & $\beta$ & $p$ & $\kappa$ & $\mu$ & $\mathbf{s}$ & $V_{50}$ & $\alpha$ for LP & $\alpha$ for HP & $p_{M}$ & $r$ \\
\hline $\mathrm{H} 1 \mathrm{~N} 1$ & $8.4 \times 10^{-9}$ & 4.6 & $2.6 \times 10^{2}$ & $2.5 \times 10^{-9}$ & 508725.2 & $3.9 \times 10^{-4}$ & $1.8 \times 10^{-2}$ & $2.2 \times 10^{-1}$ & $2.0 \times 10^{-1}$ & $2.2 \times 10^{-3}$ \\
\hline H5N1 & $6.0 \times 10^{-9}$ & 4.9 & $2.2 \times 10^{-9}$ & $9.7 \times 10^{1}$ & 372997.0 & $3.4 \times 10^{-4}$ & $5.0 \times 10^{-2}$ & $4.9 \times 10^{-1}$ & $5.4 \times 10^{-2}$ & $3.2 \times 10^{-3}$ \\
\hline
\end{tabular}

doi:10.1371/journal.pone.0150568.t003 

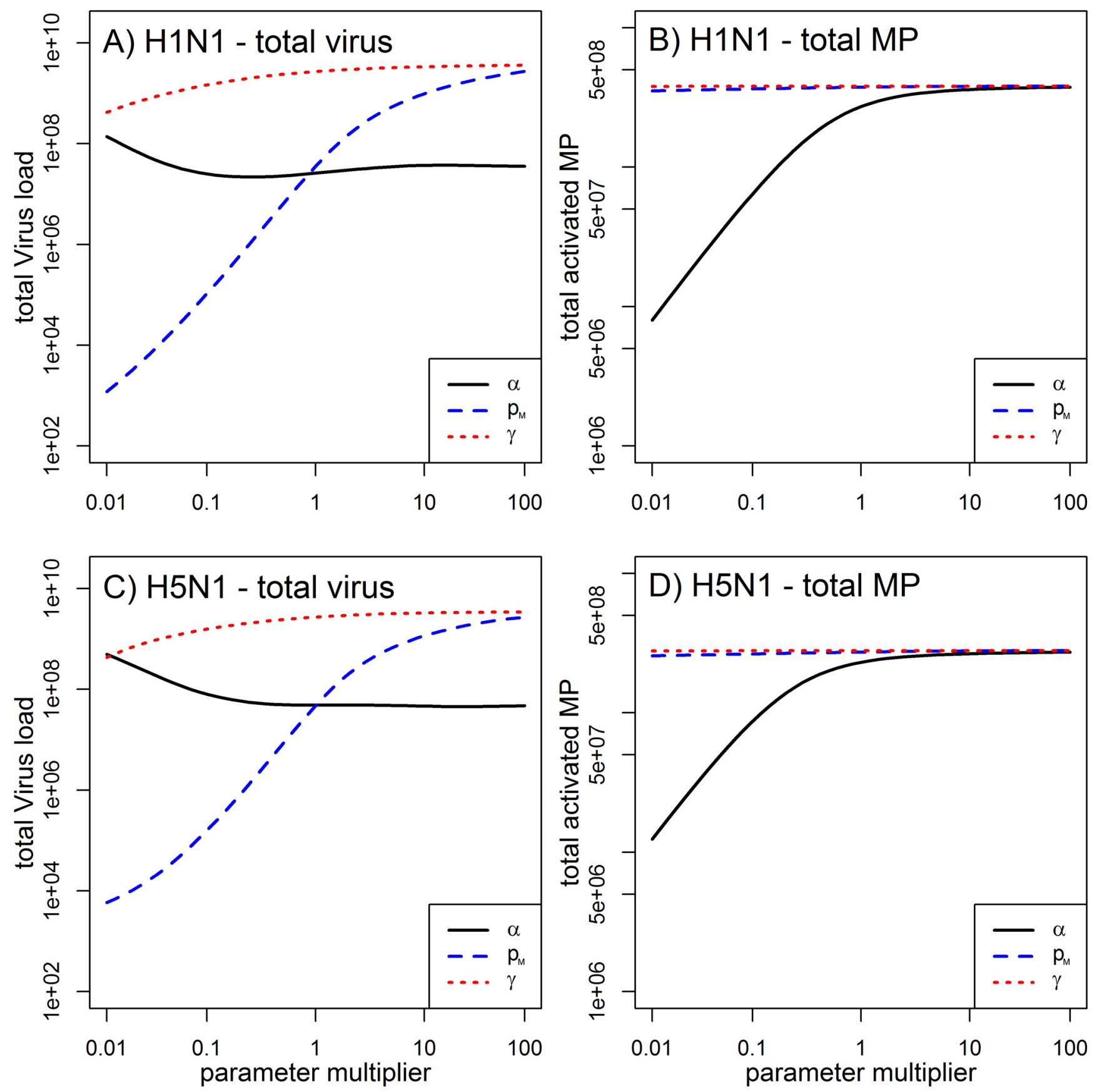

Fig 5. Impact of HP associated parameter values on total viral load and total activated macrophages. We individually varied each of the parameters $\alpha$, $p_{M}$, and $y$ in a range of $0.01-100$ times its original value for HP infections shown in Table 3 . All other parameters were kept at the values reported for the HP scenario in Tables 2 and 3.

doi:10.1371/journal.pone.0150568.g005

\section{Discussion}

Understanding why some influenza strains cause relatively little disease and pathology, while others often lead to severe outcomes, is important if we want to further improve our ability to control influenza. Here, we explored the hypothesis that macrophages, that can have both a protective effect and, through becoming infected, contribute to pathology, might be one 

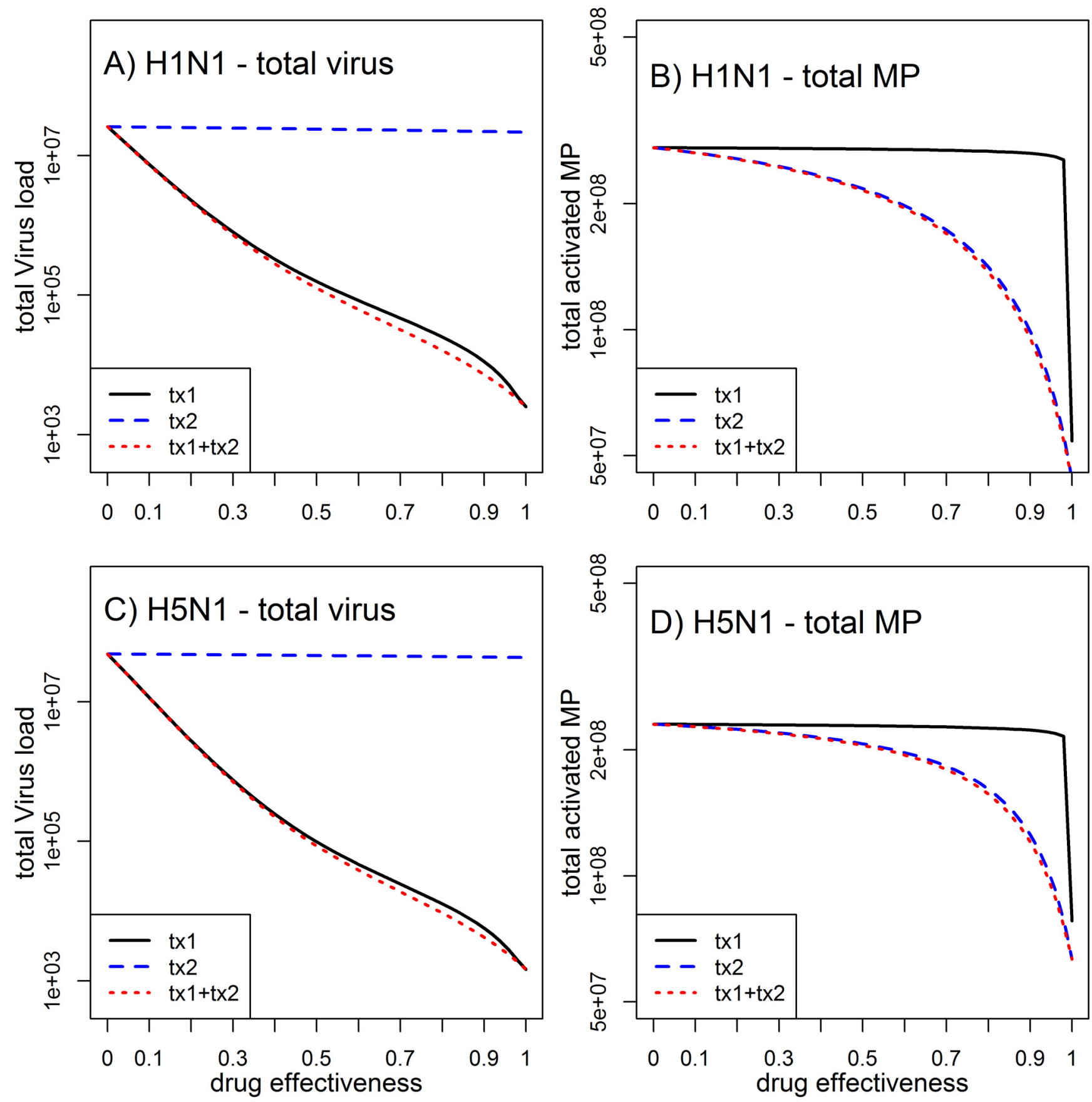

Fig 6. Impact of different treatment strategies on total viral load and total activated macrophages. Antiviral treatment (tx1), anti-inflammatory treatment (tx2) or both (tx1+tx2) were systematically varied between no effectiveness $\left(\varepsilon_{1}\right.$ and/or $\varepsilon_{2}$ at 0$)$ and $100 \%$ effectiveness $\left(\varepsilon_{1}\right.$ and/or $\varepsilon_{2}$ at 1$)$. The remaining parameters are set to the HP scenarios listed in Tables 2 and 3.

doi:10.1371/journal.pone.0150568.g006

mechanism that distinguishes LP from HP influenza infections. Macrophages are at the forefront in the defense against foreign invasion by micro-organisms. During an influenza infection, activated macrophages in the lungs stimulate cytokines, TNF $\alpha$ and TNF $\beta$, which recruit additional macrophages and other immune system cells that play a role in the recognition of foreign antigens and support proper activation of adaptive response. When macrophages 
recognize invading particles, they confront the invaders and release chemical signals which activate the immune response. Macrophages attempt to clear the virus by phagocytosis [5052]. This may be the mode by which macrophages could become infected and contribute to the total viral load during HP influenza infections.

By developing a mathematical model and fitting it to virus and macrophage data for LP and HP infections, we were able to test whether a difference in parameters associated with macrophage activation, infection, and virus production can explain observed differences between LP and $\mathrm{HP}$ virus infections. Our modeling results suggest that the productive infection of macrophages with HP influenza viruses is a plausible explanation for the different infection outcomes observed in mice infected with LP and HP virus strains. Our finding for MP contributing both to virus clearance and pathology mirrors a recent finding that dendritic cells can be a doubleedged sword in influenza infections [53].

Using our model parameterized for HP infections, we investigated the impact of antiviral and anti-inflammatory drugs. We find that while antiviral drugs can reduce virus load, the impact on pathology (which we quantified with macrophage numbers as proxy) is minimal. Some experimental studies have reported similar minimal benefits of antiviral therapies during HP influenza infection $[54,55]$.

Alternatives to antiviral drugs targeting the virus are anti-inflammatory approches. For instance, anti-TNF agents currently on the market may prove efficient in controlling the immune response [56] and thus reducing pathology during influenza infections. Our model predicted an effect of anti-inflammatory drugs in substantially lowering the number of macrophages at the site of the infection, however there was minimal impact on viral load. If both antiviral and anti-inflammatory drugs were combined, our model predicted additive effects leading to reduction in both viral load and pathology.

The usual caveats to our study apply. The fact that our model can explain the experimentally observed differences between LP and HP infections should only be taken as supportive, not confirmatory, of the idea that macrophages play such a double-edged role in virus clearance and pathology. Many other models may also adequately describe the observed data. Further, the data are from infections in mice. While influenza infections in mice capture some of the characteristics of human influenza infections, there are important differences and it is unclear what role MP play for human influenza infections.

\section{Author Contributions}

Conceived and designed the experiments: KAP AH. Performed the experiments: KAP AH. Analyzed the data: KAP DD CS AH. Contributed reagents/materials/analysis tools: KAP DD CS AH. Wrote the paper: KAP DD CS AH.

\section{References}

1. WHO. Influenza (Seasonal) Fact sheet $\mathrm{nr} 211$ : http://www.who.int/mediacentre/factsheets/fs211/en/; 2014 [March 2015]. Available from: http://www.who.int/mediacentre/factsheets/fs211/en/index.html.

2. Beigel JH, Farrar J, Han AM, Hayden FG, Hyer R, de Jong MD, et al. Avian influenza A (H5N1) infection in humans. The New England journal of medicine. 2005; 353(13):1374-85. Epub 2005/09/30. doi: 10. 1056/NEJMra052211 PMID: 16192482.

3. Smallman-Raynor M, Cliff AD. Avian influenza A (H5N1) age distribution in humans. Emerging infectious diseases. 2007; 13(3):510-2. Epub 2007/06/08. doi: 10.3201/eid1303.060849 PMID: 17552119; PubMed Central PMCID: PMCPMC2725913.

4. WHO. Human infection with avian influenza A(H7N9) virus—update: http://www.who.int/csr/don/2013 08_11/en/; 2013 [updated December 3, 2013; cited 2013 January 8 2015]. Available from: http://www. who.int/csr/don/2013_08_11/en/. 
5. Patterson KD, Pyle GF. The geography and mortality of the 1918 influenza pandemic. Bulletin of the history of medicine. 1991; 65(1):4-21. Epub 1991/01/01. PMID: 2021692.

6. Kuiken T, Rimmelzwaan G, van Riel D, van Amerongen G, Baars M, Fouchier R, et al. Avian H5N1 influenza in cats. Science. 2004; 306(5694):241. PMID: 15345779.

7. Zitzow LA, Rowe T, Morken T, Shieh WJ, Zaki S, Katz JM. Pathogenesis of avian influenza A (H5N1) viruses in ferrets. J Virol. 2002; 76(9):4420-9. PMID: 11932409.

8. Quinlivan M, Nelly M, Prendergast M, Breathnach C, Horohov D, Arkins S, et al. Pro-inflammatory and antiviral cytokine expression in vaccinated and unvaccinated horses exposed to equine influenza virus. Vaccine. 2007; 25(41):7056-64. PMID: 17825959.

9. Rimmelzwaan GF, Kuiken T, van Amerongen G, Bestebroer TM, Fouchier RA, Osterhaus AD. Pathogenesis of influenza A (H5N1) virus infection in a primate model. J Virol. 2001; 75(14):6687-91. PMID: 11413336.

10. Pawelek KA, Huynh GT, Quinlivan M, Cullinane A, Rong L, Perelson AS. Modeling within-host dynamics of influenza virus infection including immune responses. PLoS computational biology. 2012; 8(6): e1002588. Epub 2012/07/05. doi: 10.1371/journal.pcbi.1002588 PMID: 22761567; PubMed Central PMCID: PMCPMC3386161.

11. Baccam $P$, Beauchemin C, Macken CA, Hayden FG, Perelson AS. Kinetics of influenza A virus infection in humans. J Virol. 2006; 80(15):7590-9. PMID: 16840338.

12. Handel A, Longini IM Jr., Antia R. Towards a quantitative understanding of the within-host dynamics of influenza A infections. Journal of the Royal Society, Interface / the Royal Society. 2010; 7(42):35-47. Epub 2009/05/29. doi: 10.1098/rsif.2009.0067 PMID: 19474085; PubMed Central PMCID: PMCPMC2839376.

13. Handel A, Longini IM Jr., Antia R. Neuraminidase inhibitor resistance in influenza: assessing the danger of its generation and spread. PLoS Comput Biol. 2007; 3(12):e240. PMID: 18069885.

14. Smith AM, Adler FR, Perelson AS. An accurate two-phase approximate solution to an acute viral infection model. J Math Biol. 2010; 60(5):711-26. PMID: 19633852. doi: 10.1007/s00285-009-0281-8

15. Hancioglu B, Swigon D, Clermont G. A dynamical model of human immune response to influenza $A$ virus infection. J Theor Biol. 2007; 246(1):70-86. PMID: 17266989.

16. Bocharov GA, Romanyukha AA. Mathematical model of antiviral immune response. III. Influenza A virus infection. J Theor Biol. 1994; 167(4):323-60. PMID: 7516024.

17. Rimmelzwaan GF, Baars M, Claas EC, Osterhaus AD. Comparison of RNA hybridization, hemagglutination assay, titration of infectious virus and immunofluorescence as methods for monitoring influenza virus replication in vitro. J Virol Methods. 1998; 74(1):57-66. Epub 1998/10/08. S0166-0934(98)000718 [pii]. PMID: 9763129.

18. Smith AM, Ribeiro RM. Modeling the viral dynamics of influenza A virus infection. Crit Rev Immunol. 2010; 30(3):291-8. Epub 2010/04/08. 1629d2c3507c27c5,5aa3a295481caaac [pii]. PMID: 20370636.

19. Miao H, Hollenbaugh JA, Zand MS, Holden-Wiltse J, Mosmann TR, Perelson AS, et al. Quantifying the early immune response and adaptive immune response kinetics in mice infected with influenza $A$ virus. Journal of virology. 2010; 84(13):6687-98. Epub 2010/04/23. doi: 10.1128/jvi.00266-10 PMID: 20410284; PubMed Central PMCID: PMCPMC2903284.

20. Beauchemin CA, Handel A. A review of mathematical models of influenza A infections within a host or cell culture: lessons learned and challenges ahead. BMC Public Health. 2011; 11 Suppl 1:S7. Epub 2011/03/05. 1471-2458-11-S1-S7 [pii] PMID: 21356136.

21. Saenz RA, Quinlivan M, Elton D, Macrae S, Blunden AS, Mumford JA, et al. Dynamics of influenza virus infection and pathology. Journal of virology. 2010; 84(8):3974-83. Epub 2010/02/05. JVI.0207809 [pii] doi: 10.1128/JVI.02078-09 PMID: 20130053.

22. Dobrovolny HM, Baron MJ, Gieschke R, Davies BE, Jumbe NL, Beauchemin CA. Exploring cell tropism as a possible contributor to influenza infection severity. PloS one. 2010; 5(11):e13811. Epub 2010/12/ 03. doi: 10.1371/journal.pone.0013811 PMID: 21124892; PubMed Central PMCID: PMCPMC2990709.

23. Hofmann P, Sprenger H, Kaufmann A, Bender A, Hasse C, Nain M, et al. Susceptibility of mononuclear phagocytes to influenza $A$ virus infection and possible role in the antiviral response. Journal of leukocyte biology. 1997; 61(4):408-14. Epub 1997/04/01. PMID: 9103226.

24. Elhelu MA. The role of macrophages in immunology. Journal of the National Medical Association. 1983; 75(3):314-7. Epub 1983/03/01. PMID: 6343621; PubMed Central PMCID: PMCPMC2561478.

25. Martinez FO, Sica A, Mantovani A, Locati M. Macrophage activation and polarization. Frontiers in bioscience: a journal and virtual library. 2008; 13:453-61. Epub 2007/11/06. PMID: 17981560.

26. Janeway AC Jr, Travers P, Walport M, Shlomchik MJ. Immunobiology. 5 ed. New York: Garland Science; 2001. 
27. Schneider C, Nobs SP, Heer AK, Kurrer M, Klinke G, van Rooijen N, et al. Alveolar macrophages are essential for protection from respiratory failure and associated morbidity following influenza virus infection. PLoS pathogens. 2014; 10(4):e1004053. Epub 2014/04/05. doi: 10.1371/journal.ppat.1004053 PMID: 24699679; PubMed Central PMCID: PMCPMC3974877.

28. Perrone LA, Plowden JK, Garcia-Sastre A, Katz JM, Tumpey TM. H5N1 and 1918 pandemic influenza virus infection results in early and excessive infiltration of macrophages and neutrophils in the lungs of mice. PLoS pathogens. 2008; 4(8):e1000115. Epub 2008/08/02. doi: 10.1371/journal.ppat.1000115 PMID: 18670648; PubMed Central PMCID: PMCPMC2483250.

29. Yu WC, Chan RW, Wang J, Travanty EA, Nicholls JM, Peiris JS, et al. Viral replication and innate host responses in primary human alveolar epithelial cells and alveolar macrophages infected with influenza H5N1 and H1N1 viruses. Journal of virology. 2011; 85(14):6844-55. Epub 2011/05/06. doi: 10.1128/jvi. 02200-10 PMID: 21543489; PubMed Central PMCID: PMCPMC3126566.

30. Sakabe S, Iwatsuki-Horimoto K, Takano R, Nidom CA, Le M, Nagamura-Inoue T, et al. Cytokine production by primary human macrophages infected with highly pathogenic $\mathrm{H} 5 \mathrm{~N} 1$ or pandemic $\mathrm{H} 1 \mathrm{~N} 1$ 2009 influenza viruses. The Journal of general virology. 2011; 92(Pt 6):1428-34. Epub 2011/03/04. doi: 10.1099/vir.0.030346-0 PMID: 21367984; PubMed Central PMCID: PMCPMC3168279.

31. Smith AM, McCullers JA, Adler FR. Mathematical model of a three-stage innate immune response to a pneumococcal lung infection. Journal of theoretical biology. 2011; 276(1):106-16. Epub 2011/02/09. doi: 10.1016/j.jtbi.2011.01.052 PMID: 21300073; PubMed Central PMCID: PMCPMC3066295.

32. Murphy K. Janeway's Immunobiology. 8th ed: Garland Science; 2012.

33. Moscona A. Neuraminidase inhibitors for influenza. The New England journal of medicine. 2005; 353 (13):1363-73. Epub 2005/09/30. doi: 10.1056/NEJMra050740 PMID: 16192481.

34. Dobrovolny HM, Gieschke R, Davies BE, Jumbe NL, Beauchemin CA. Neuraminidase inhibitors for treatment of human and avian strain influenza: A comparative modeling study. Journal of theoretical biology. 2011; 269(1):234-44. Epub 2010/10/26. doi: 10.1016/j.jtbi.2010.10.017 PMID: 20970433.

35. Julkunen I, Melen K, Nyqvist M, Pirhonen J, Sareneva T, Matikainen S. Inflammatory responses in influenza A virus infection. Vaccine. 2000; 19 Suppl 1:S32-7. S0264410X00002759 [pii]. PMID: 11163460.

36. Jefferson $T$, Jones MA, Doshi P, Del Mar CB, Hama R, Thompson MJ, et al. Neuraminidase inhibitors for preventing and treating influenza in healthy adults and children. The Cochrane database of systematic reviews. 2014; 4:CD008965. Epub 2014/04/11. doi: 10.1002/14651858.CD008965.pub4 PMID: 24718923.

37. Johnson SG. The NLopt nonlinear-optimization package.

38. Soetaert K, Petzoldt T, Setzer RW. Solving Differential Equations in R: Package deSolve. Journal of Statistical Software. 2010; 33(9):1-25.

39. Wickham H, Francois R. A Grammar of Data Manipulation. 2015.

40. Wickham H. ggplot2: Elegant Graphics for Data Analysis: Springer-Verlag New York; 2009.

41. Sanders CJ, Vogel P, McClaren JL, Bajracharya R, Doherty PC, Thomas PG. Compromised respiratory function in lethal influenza infection is characterized by the depletion of type I alveolar epithelial cells beyond threshold levels. American journal of physiology Lung cellular and molecular physiology. 2013; 304(7):L481-8. Epub 2013/01/29. doi: 10.1152/ajplung.00343.2012 PMID: 23355384; PubMed Central PMCID: PMCPMC3627938.

42. Smith AM, Perelson AS. Influenza A virus infection kinetics: quantitative data and models. Wiley Interdiscip Rev Syst Biol Med. 2011; 3(4):429-45. Epub 2011/01/05. doi: 10.1002/wsbm.129 PMID: 21197654.

43. Handel A, Brown J, Stallknecht D, Rohani P. A multi-scale analysis of influenza A virus fitness tradeoffs due to temperature-dependent virus persistence. PLoS computational biology. 2013; 9(3): e1002989. Epub 2013/04/05. doi: 10.1371/journal.pcbi.1002989 PMID: 23555223; PubMed Central PMCID: PMCPMC3605121.

44. Handel A, Akin V, Pilyugin SS, Zarnitsyna V, Antia R. How sticky should a virus be? The impact of virus binding and release on transmission fitness using influenza as an example. Journal of the Royal Society, Interface / the Royal Society. 2014; 11(92):20131083. Epub 2014/01/17. doi: 10.1098/rsif.2013. 1083 PMID: 24430126; PubMed Central PMCID: PMCPMC3899878.

45. Dobson J, Whitley RJ, Pocock S, Monto AS. Oseltamivir treatment for influenza in adults: a meta-analysis of randomised controlled trials. Lancet (London, England). 2015; 385(9979):1729-37. Epub 2015/ 02/03. doi: 10.1016/s0140-6736(14)62449-1 PMID: 25640810.

46. Muthuri SG, Venkatesan S, Myles PR, Leonardi-Bee J, Al Khuwaitir TS, Al Mamun A, et al. Effectiveness of neuraminidase inhibitors in reducing mortality in patients admitted to hospital with influenza $A$ H1N1pdm09 virus infection: a meta-analysis of individual participant data. The Lancet Respiratory 
medicine. 2014; 2(5):395-404. Epub 2014/05/13. doi: 10.1016/s2213-2600(14)70041-4 PMID: 24815805.

47. Ebell MH, Call M, Shinholser J. Effectiveness of oseltamivir in adults: a meta-analysis of published and unpublished clinical trials. Family practice. 2013; 30(2):125-33. Epub 2012/09/22. doi: 10.1093/ fampra/cms059 PMID: 22997224.

48. White NJ, Webster RG, Govorkova EA, Uyeki TM. What is the optimal therapy for patients with H5N1 influenza? PLoS medicine. 2009; 6(6):e1000091. Epub 2009/06/26. doi: 10.1371/journal.pmed. 1000091 PMID: 19554084; PubMed Central PMCID: PMCPMC2694988.

49. Zheng BJ, Chan KW, Lin YP, Zhao GY, Chan C, Zhang HJ, et al. Delayed antiviral plus immunomodulator treatment still reduces mortality in mice infected by high inoculum of influenza $A / H 5 N 1$ virus. Proceedings of the National Academy of Sciences of the United States of America. 2008; 105(23):8091-6. Epub 2008/06/05. doi: 10.1073/pnas.0711942105 PMID: 18523003; PubMed Central PMCID: PMCPMC2430364.

50. Baskin CR, Bielefeldt-Ohmann H, Tumpey TM, Sabourin PJ, Long JP, Garcia-Sastre A, et al. Early and sustained innate immune response defines pathology and death in nonhuman primates infected by highly pathogenic influenza virus. Proceedings of the National Academy of Sciences of the United States of America. 2009; 106(9):3455-60. Epub 2009/02/17. doi: 10.1073/pnas.0813234106 PMID: 19218453; PubMed Central PMCID: PMCPMC2642661.

51. Murray PJ, Wynn TA. Protective and pathogenic functions of macrophage subsets. Nat Rev Immunol. 2011; 11(11):723-37. Epub 2011/10/15. nri3073 [pii] doi: 10.1038/nri3073 PMID: 21997792.

52. Mosser DM, Edwards JP. Exploring the full spectrum of macrophage activation. Nat Rev Immunol. 2008; 8(12):958-69. Epub 2008/11/26. nri2448 [pii] doi: 10.1038/nri2448 PMID: 19029990.

53. Aldridge JR Jr., Moseley CE, Boltz DA, Negovetich NJ, Reynolds C, Franks J, et al. TNF/iNOS-producing dendritic cells are the necessary evil of lethal influenza virus infection. Proceedings of the National Academy of Sciences of the United States of America. 2009; 106(13):5306-11. Epub 2009/03/13. doi: 10.1073/pnas.0900655106 PMID: 19279209; PubMed Central PMCID: PMCPMC2664048.

54. Govorkova EA, Baranovich T, Seiler P, Armstrong J, Burnham A, Guan Y, et al. Antiviral resistance among highly pathogenic influenza A (H5N1) viruses isolated worldwide in 2002-2012 shows need for continued monitoring. Antiviral research. 2013; 98(2):297-304. Epub 2013/03/06. doi: 10.1016/j. antiviral.2013.02.013 PMID: 23458714; PubMed Central PMCID: PMCPMC3648604.

55. Belser JA, Szretter KJ, Katz JM, Tumpey TM. Simvastatin and oseltamivir combination therapy does not improve the effectiveness of oseltamivir alone following highly pathogenic avian H5N1 influenza virus infection in mice. Virology. 2013; 439(1):42-6. Epub 2013/03/05. doi: 10.1016/j.virol.2013.01.017 PMID: 23453580.

56. Palladino MA, Bahjat FR, Theodorakis EA, Moldawer LL. Anti-TNF-alpha therapies: the next generation. Nature reviews Drug discovery. 2003; 2(9):736-46. Epub 2003/09/03. doi: 10.1038/nrd1175 PMID: 12951580 